\title{
Efeito da unidade de resfriamento e conservação, do tempo de armazenamento e diluidor sobre as características do sêmen de cães
}

\section{Effect of the chilling sistems, time of conservation and extender on dog's semen characteristics}

\author{
Marco Antônio Rocha Ferreira, ${ }^{*}$ Bianca Perez Barcelos, ${ }^{*}$ Luciano Jamas, ${ }^{*}$ \\ Felipe Zandonadi Brandão, ${ }^{\star * *}$ Luiz Altamiro Garcia Nogueira, ${ }^{\star \star *}$ Tânia Góes de Pinho***
}

\begin{abstract}
Resumo
O presente estudo teve como objetivo determinar o efeito de dois diferentes sistemas de resfriamento (Equitainer ${ }^{\circledR}$ II e caixa isotérmica comercial) sobre as características do sêmen de cães após 12 e 24 horas de conservação em dois meios diluidores : meio mínima contaminação e meio lactose-gema modificado. Foram utilizados 15 ejaculados de cinco cães, que foram avaliados quanto à motilidade progressiva, vigor espermático, patologia espermática, porcentagem de espermatozóides vivos e com membrana plasmática íntegra. As amostras foram diluídas na proporção de 1:3 nos meios de conservação, armazenadas nos dois sistemas de transporte e analisadas após 12 e 24 horas de resfriamento. Os resultados obtidos mostram que não houve diferença significativa de nenhuma das características seminais das amostras conservadas no Equitainer ${ }^{\oplus}$ || e caixa isotérmica comercial. Verifica-se que após 24 horas de armazenamento houve um declínio significativo $(P<0,05)$ de todas as características do sêmen independentemente dos meios ou unidades de conservação. Porém, após 12 horas de armazenamento, não houve diferença significativa da motilidade progressiva, vigor espermático, porcentagem de espermatozóides vivos e patologia espermática entre o sêmen fresco e o conservado em lactose gema modificado, independentemente da unidade de conservação utilizada. O mesmo não ocorreu no sêmen conservado no mínima contaminação, cujas características, neste momento, foram diferentes $(P<0,05)$ daquelas observadas no sêmen fresco, com exceção da patologia espermática. A integridade da membrana plasmática foi a única característica que apresentou diferença significativa $(P<0,05)$ entre o sêmen fresco e o conservado por 12 horas no meio lactose gema modificado. Conclui-se que o tipo de unidade de resfriamento utilizado não influenciou as características seminais durante o período estudado, e que o tempo de 24 horas sob resfriamento influencia consideravelmente as características do sêmen, mas que após 12 e 24 horas de conservação, o meio lactose gema foi superior ao mínima contaminação na manutenção da maioria dos parâmetros do sêmen.
\end{abstract}

Palavras-chave: sêmen, cães, resfriamento, diluidores, unidades de resfriamento/conservação.

\begin{abstract}
The present study purposed to compare the effect of two different chilled systems (Equitainer ${ }^{\circledR} \|$ and icefoam box) and two different diluents (minimal contamination and modified lactose yolk extender), on the dog's seminal characteristics after 12 and 24 hours of conservation. Fifteen ejaculates (three of each dog) were analyzed for sperm motility, vigor, sperm defects, live spermatozoa percentage and sperm membrane integrity percentage. Samples were diluted 1:3 using both extenders. They were storied in two kinds of chilling systems and were analyzed after 12 and 24 hours of conservation. The results showed that there was no statistic difference between any characteristics of the semen conserved into Equitainer ${ }^{\circledast} \mathrm{II}$ and icefoam box. After 24 hours of conservation there was a significant effect $(P<0.05)$ in all semen characteristics independent of any kind of extender or containers. But there was no significant difference in the sperm motility, vigor, sperm morphology and in percentage of live spermatozoa between fresh and the 12 hours in laktose yolk conserved semen's. The same was not observed in semen conserved in the minimal contamination extender that showed significant differences $(P<0.05)$ for all studied characteristics, except total sperm defect. Percentages of sperm membrane integrity were the only semen characteristic different $(P<0.05)$ between fresh and the 12 hours in lactose yolk conserved semen's. It can be conclude that the kind of used container didn't affect the semen characteristics. The time (24 hours) of conservation affected considerably the semen quality. After 12 and 24 hours of conservation the modified lactose yolk extender maintained semen parameters better than minimal contamination extender.
\end{abstract}

Keywords: semen, dogs, chilling, extenders, chilled systems.

\footnotetext{
*Alunos do Programa de Pós-Graduação em Medicina Veterinária - FV/UFF.

**Polícia Militar do Estado do Rio de Janeiro.

${ }^{* * *}$ Professores da Faculdade de Veterinária da Universidade Federal Fluminense.
} 


\section{Introdução}

O objetivo de se resfriar o sêmen é diminuir o metabolismo dos espermatozóides durante sua conservação, prolongando a sua vida útil (England e Ponzio, 1996). Os dados da literatura quanto à fertilidade do sêmen resfriado de cães ainda são escassos (Linde-Forsberg, 1995), mas os relatos mostram que as taxas de concepção podem ser semelhantes às da inseminação artificial (IA) utilizando sêmen fresco (Silva et al., 2004).

Para que o resfriamento do sêmen alcance êxito, deve-se levar em consideração vários fatores, como: a escolha dos diluidores, taxa de diluição adequada, curva de resfriamento e manutenção de uma temperatura específica durante o armazenamento (Almeida, 1998). Quando associados, estes fatores reduzem o metabolismo dos espermatozóides, minimizam as lesões de membrana plasmática, evitando a perda precoce da longevidade (Loomis, 1992; Farstad, 1996). Linde-Forsberg (1995) constatou que o sêmen de cães, diluído e resfriado, e de boa qualidade, reteve sua capacidade fertilizante por pelo menos 12 a 24 horas, tempo este que permite o seu transporte.

Os diluidores utilizados para o resfriamento e congelamento de sêmen de cães foram adaptados de outras espécies, sendo os mais empregados os meios à base de leite desnatado, lactose-gema, citrato-gema e tris-gema (England e Ponzio, 1996, Magnago, 2000). Devido a sua composição e a capacidade de tamponamento, os diluidores à base de leite têm sido amplamente utilizados.

A taxa de resfriamento do sêmen tem fundamental importância na preservação do espermatozóide (Amann e Grahan, 1993). Curvas apropriadas de resfriamento minimizam o choque térmico e podem permitir o armazenamento de espermatozóides por um longo período (Lagares et al., 2000). Quanto mais baixa a temperatura de armazenamento, mais lento deve ser o resfriamento (Pickett, 1993). As curvas rápidas de resfriamento podem causar danos irreversíveis aos espermatozóides (Watson, 1981).

Atualmente existem unidades de resfriamento e transporte, de origem japonesa (Nishikawa e Wide, 1949), francesa (Palmer,1974), holandesa (Van Der Holst, 1984), norteamericana (Douglas-Hamilton et al., 1984), alemã (Hueck, 1990) e brasileira (Silva Filho, 1994). A mais difundida mundialmente, inclusive no Brasil, é a norte-americana, que utiliza o Equitainer ${ }^{\circledR}$ (Valle, 2001).

Assim, o objetivo do presente experimento foi determinar os efeitos de dois sistemas de resfriamento e conservação (Equitainer ${ }^{\circledR}$ II e caixa isotérmica comercial) e de dois diluidores (meio mínima contaminação - Kenney et al., 1975 e lactose-gema de ovo modificado - Silva Filho, 1994) sobre as características do sêmen de cães durante 24 horas de resfriamento.

\section{Material e métodos}

Foram utilizados cinco cães adultos hígidos de diferentes raças, mantidos no canil da Polícia Militar do Estado do Rio de Janeiro. Foram obtidas três amostras de sêmen por animal com intervalos de três dias, através de manipulação digital do pênis e prepúcio (Feldman e Nelson, 1996), sendo utilizada apenas a segunda fração seminal (espermática) e parte da terceira, com a finalidade de se obter um volume mínimo necessário para a posterior diluição do sêmen.

$\mathrm{O}$ sêmen foi então mantido em banho-maria a $37^{\circ} \mathrm{C}$, até o momento do resfriamento, quando cada ejaculado foi diluído na proporção de 1:3 em dois diferentes meios diluidores: meio mínima contaminação (MMC) e lactose-gema modificado (LGM) e submetidos a resfriamento em dois ambientes distintos: Equitainer ${ }^{\circledR}$ II e caixa isotérmica simples $(27 \times 18 \times$ $18 \mathrm{~cm}$ ) contendo $1000 \mathrm{~mL}$ de gelo biológico (as amostras foram acondicionadas a $10 \mathrm{~cm}$ da fonte de frio e o espaço restante da caixa isotérmica preenchida com folhas de jornal), por um período de 12 e 24 horas.

As amostras de sêmen foram avaliadas quanto à motilidade progressiva, vigor (CBRA, 1996), patologia espermática (Blom, 1973), percentual de espermatozóides vivos (utilizando o corante eosina) e integridade da membrana plasmática, determinada através da realização do teste hiposmótico $(\mathrm{HO})$ (Kumi-Diaka, 1993), no sêmen fresco e no conservado por 12 e 24 horas. Apenas motilidade e vigor foram avaliados no tempo zero e após a diluição.

Para determinar a temperatura no interior da caixa isotérmica e assim confeccionar a curva de resfriamento, foi utilizado um termômetro digital de leitura, sendo este procedimento repetido em três momentos distintos ao longo do experimento. Um sensor foi colocado no interior da caixa, imerso em um tubo de $12 \mathrm{~mL}$ contendo água destilada a $37^{\circ} \mathrm{C}$, e a temperatura foi monitorada 30 minutos após o fechamento da caixa e, em seguida, a cada 60 minutos até completar 12 horas de conservação. Após este período, a temperatura foi aferida decorridas 24 horas do fechamento da caixa. Para o Equitainer $^{\oplus} \mathrm{II}$, foi considerada a curva de resfriamento informada pelo fabricante.

$\mathrm{Na}$ análise estatística todas as características seminais oriundas dos 15 ejaculados foram avaliadas em relação ao tipo de meio, tempo de conservação e unidade de transporte. Para tal, foi utilizada análise de variância (ANOVA) e o teste SNK do Software GraphPad Instat 3.0532 bit for Win 95/NT. O mesmo sistema estatístico foi usado na obtenção das médias e desvios-padrão. As diferenças foram consideradas significativas quando $\mathrm{P}<0,05$.

\section{Resultados e discussão}

As características do sêmen de cães avaliadas logo após a coleta encontram-se na Tabela 1. A motilidade progressiva média $(89,66 \pm 4,11)$ foi mais elevada do que a observada por Cunha (1997), que obteve $74,4 \%$ e foram semelhantes às de Rota (1995) e England e Ponzio (1996) que encontraram respectivamente valores de $78,6 \%$ e 85,0 a $95,0 \%$. O vigor do sêmen está de acordo com os observados por Magnago (2000) e Cardoso (2002) que encontraram respectivamente 4,8 e 4,9, sendo superior ao relatado por Almeida (1998). Entretanto, é importante ressaltar que essas características (motilidade e vigor), são parâmetros subjetivos, podendo determinar estas diferenças observadas entre os diferentes autores.

A porcentagem de espermatozóides vivos, no sêmen fresco, foi de $90,8 \pm 3,0 \%$ e variou de $84,0 \%$ a $96,0 \%$. Os resultados foram semelhantes aos relatados por Almeida (1998) $(91,7 \%)$ e superiores aos de Castro (2003) que verificou o valor de $83,6 \%$. As porcentagens de espermatozóides com membrana 
plasmática íntegra foram semelhantes aos de Castro (2003), que observou valores de $83,6 \%$ e foram pouco inferiores aos descritos por England e Ponzio (1996) que relataram 89,0\% a 96,0\% de espermatozóides com membrana íntegra.

Não houve diferença $(P>0,05)$ entre as características de motilidade e vigor do sêmen fresco e do diluído no tempo zero.

A curva de resfriamento do sêmen conservado na caixa isotérmica baseada nas médias das temperaturas verificadas
Após 30 minutos, a temperatura foi de $9,1 \pm 0,3^{\circ} \mathrm{C}$ e de $9,3 \pm$ $0,3^{\circ} \mathrm{C}$ após 1 hora. A queda de temperatura mensurada nesse período foi de $28,0^{\circ} \mathrm{C}$, podendo-se estimar que houve um decréscimo aproximado de $0,9^{\circ} \mathrm{C}$ por minuto.

A queda de temperatura verificada nos primeiros 30 minutos de resfriamento foi acima dos 0,3 a $0,5^{\circ} \mathrm{C} / \mathrm{min}$ recomendados por Douglas - Hamilton et al. (1984) e presente no Equitainer para minimizar o choque térmico e, conseqüentemente, os danos aos espermatozóides na espécie eqüina.

Sabe-se que o tipo de conservação, a curva de resfriamento e a estabilidade da temperatura influem diretamente nas características do sêmen. Durante o processo de resfriamento ocorre o choque térmico e a formação de componentes tóxicos no meio diluidor utilizado, que determinam lesões na membrana plasmática e/ou mesmo a morte dos espermatozóides (Magnago, 2000). Apesar da queda de temperatura na caixa isotérmica ter sido mais acentuada do que a desejada, não foi observada influência $(P>0,05)$ da unidade de conservação sobre as características seminais, sendo os resultados observados na caixa isotérmica semeIhantes àqueles verificados no sêmen conservado em Equitainer® II, tanto após 12 horas quanto após 24 horas de resfriamento e independentemente do meio diluidor utilizado (Tabela 1). nas primeiras 12 horas e as 24 horas de incubação, encontrase na Figura 1.

A temperatura média no interior da caixa isotérmica, no início do processo de conservação das amostras foi de $37,1 \pm 0,1^{\circ} \mathrm{C}$.

Tabela 1: Características do sêmen de cães diluído nos meios Lactose Gema Modificado (LGM) e Meio Mínima Contaminação (MMC), conservados por 12 e 24 horas em relação à unidade de transporte (Equitainer ${ }^{\circledR}$ II e caixa isotérmica) (média \pm desvio-padrão).

\begin{tabular}{|c|c|c|c|c|c|c|}
\hline & $\begin{array}{c}\text { Características seminais } \\
\text { Tempo }\end{array}$ & Fresco & \multicolumn{2}{|l|}{ LGM } & \multicolumn{2}{|l|}{$M M C$} \\
\hline \multirow{4}{*}{ 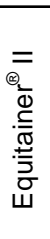 } & Motilidade Progressiva (\%) & $89,66 \pm 4,41^{a}$ & $71,00 \pm 12,13^{\mathrm{abA}}$ & $57,33 \pm 25,97^{\mathrm{DA}}$ & $44,33 \pm 29,93^{\mathrm{CA}}$ & $24,33 \pm 26,31^{\mathrm{CA}}$ \\
\hline & Vigor Espermático $(0-5)$ & $4,83 \pm 0,36^{a}$ & $3,46 \pm 0,66^{\mathrm{abcB}}$ & $2,73 \pm 1,25^{\mathrm{bdB}}$ & $2,43 \pm 1,25^{\mathrm{ceB}}$ & $1,60 \pm 1,44^{\mathrm{deB}}$ \\
\hline & Espermatozóides Vivos (\%) & $90,80 \pm 3,00^{a}$ & $81,20 \pm 6,01^{\mathrm{abcc}}$ & $69,73 \pm 17,26^{\mathrm{bdC}}$ & $66,40 \pm 19,67^{\mathrm{ceC}}$ & $53,46 \pm 20,10^{\mathrm{dec}}$ \\
\hline & Memb. Plasmática Íntegra (\%) & $86,86 \pm 3,44^{a}$ & $75,93 \pm 7,85^{\mathrm{bcdD}}$ & $68,46 \pm 16,38^{\mathrm{CeD}}$ & $70,26 \pm 5,04^{\mathrm{dfD}}$ & $62,33 \pm 8,65^{\mathrm{efD}}$ \\
\hline \multirow{4}{*}{ 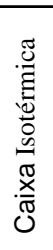 } & Motilidade Progressiva (\%) & $89,66 \pm 4,41^{\mathrm{a}}$ & $67,00 \pm 18,59^{\mathrm{abA}}$ & $46,66 \pm 29,86^{\mathrm{bA}}$ & $42,00 \pm 29,80^{\mathrm{CA}}$ & $20,73 \pm 26,59^{\mathrm{cA}}$ \\
\hline & Vigor Espermático (0 - 5) & $4,83 \pm 0,36^{a}$ & $3,33 \pm 0,69^{a b c B}$ & $2,50 \pm 1,39^{\mathrm{bB}}$ & $2,36 \pm 1,20^{\mathrm{ceB}}$ & $1,30 \pm 1,53^{\mathrm{deB}}$ \\
\hline & Espermatozóides Vivos (\%) & $90,80 \pm 3,00^{\mathrm{a}}$ & $77,73 \pm 7,50^{\mathrm{abcc}}$ & $65,60 \pm 19,58^{\mathrm{bdc}}$ & $69,20 \pm 18,06^{\mathrm{ceC}}$ & $51,00 \pm 26,01^{\mathrm{dec}}$ \\
\hline & Memb. Plasmática Íntegra (\%) & $86,86 \pm 3,44^{\mathrm{a}}$ & $74,13 \pm 7,51^{\mathrm{bcdD}}$ & $64,13 \pm 8,03^{\mathrm{ceD}}$ & $69,13 \pm 4,81^{\mathrm{dfD}}$ & $59,86 \pm 13,62^{\mathrm{efD}}$ \\
\hline
\end{tabular}


Considerando-se o tempo de conservação e meio diluidor utilizado, verifica-se que as características do sêmen resfriado por 12 horas em LGM foram semelhantes àquelas observadas no sêmen fresco, exceto para a integridade de membrana; porém, após 24 horas, todas as características estudadas foram diferentes $(P<0,05)$.

Magnago (2000) trabalhando com o diluidor de mínima contaminação, também encontrou diferença na motilidade progressiva e vigor entre o sêmen fresco e o conservado por 22 horas, diferente de Cunha (1997) que não observou tal diferença utilizando meio glicina-gema após 24 horas de resfriamento.

As características do sêmen diluído em MMC após 12 e 24 horas de conservação foram diferentes daquelas observadas no sêmen fresco, com exceção da patologia espermática total que foi semelhante após 12 horas de conservação. Esses resultados são diferentes dos de Cunha (1997) e Almeida (1998) que não encontraram diferença significativa na motilidade progressiva e vigor do sêmen a fresco e do conservado por 24 horas sob resfriamento em meio à base de leite desnatado e glicose. Observa-se um aumento na temperatura após a abertura da caixa isotérmica. Tal fato pode justificar os resultados obtidos.

Verifica-se que o meio LGM foi mais eficaz na conservação das características seminais após 12 horas de resfriamento, independentemente da unidade de conservação utilizada. Apesar do resfriamento causar um estresse térmico ao espermatozóide, o meio contendo gema de ovo proporcionou uma maior capacidade protetora quanto aos danos causados pelo choque térmico, o que foi também observado por Foote e Leonard (1964) e Cunha (1997). Ainda, segundo England e Allen (1992), a concentração de lactose presente no meio LGM

\section{Referências}

ALMEIDA, L. E. F. Viabilidade espermática do sêmen de cães, nas 24 e 48 horas após a diluição e resfriamento em container para transporte. Niterói, 1998, 40 p. Dissertação (Mestrado em Medicina Veterinária) Faculdade de Veterinária, Universidade Federal Fluminense, Niterói, 1998.

AMANN, R. P., GRAHAN, J. K. Spermatozoa function. In: MCKINNON, A. O., VOSS, J. L. Equine Reproduction. p. 715-745. 1993.

BLOM, E. The ultrastructure of some characteristic sperm defects and a proposal for a new classification of the bull spermogram. Nord. Vet. Med., v. 25, n. 7, p. 383-391, 1973.

BOUCHARD, G. F., MORRIS, J. K., SIKES, J. D. et al. Effect of storage temperature, cooling rates and two different semen extenders on canine spermatozoa motility. Theriogenology, v. 34, n. 1, p. 147-157, 1990.

CARDOSO, R. C. S. Criopreservação de sêmen canino com um diluidor à base de água de coco. Ciência Rural, v. 32, n. 4, p. 657-661. 2002. CASTRO, A. C. N. Comparação entre os crioprotetores glicerol e etilenoglicol na congelação do sêmen canino. Niterói, 2003, 69 p. Dissertação (Mestrado em Medicina Veterinária) - Faculdade de Veterinária, Universidade Federal Fluminense, Niterói, 2003.

COLÉGIO Brasileiro de Reprodução Animal (CBRA). Manual para exame andrológico e avaliação de sêmen animal. 2. ed. Belo Horizonte, 49 p. 1998.

CUNHA, I. C. N. Estudo da viabilidade do processo de refrigeração do sêmen canino à base de leite e glicina gema. Botucatu, 124 p. Dissertação (Mestrado em Reprodução Animal) - Faculdade de Medicina Veterinária e Zootecnia, Universidade Estadual Paulista, Botucatu, São Paulo, 1997. funciona como solução tampão, retirando íons hidrogênio do meio e permitindo assim uma melhor conservação do sêmen. $\mathrm{O}$ fato de o diluidor MMC apresentar uma queda mais rápida da motilidade pode ser devido à sua menor capacidade tamponante (Cunha, 1997).

Nota-se que a conservação do sêmen por 24 horas influenciou consideravelmente todas as características estudadas, sendo que o aumento da porcentagem de defeitos totais após ocorreu, principalmente, devido à elevação do índice de espermatozóides com cauda dobrada, causado possivelmente pelo tempo de estocagem a baixas temperaturas.

A integridade da membrana plasmática foi a característica mais influenciada pela conservação do sêmen sob resfriamento, sendo a única que apresentou diferença $(P<0,05)$ entre o sêmen fresco e o conservado por 12 horas no meio LGM. De fato, a determinação da integridade da membrana plasmática tem sido indicada na avaliação do sêmen devido a sua alta correlação com a capacidade fecundante do mesmo (Jeyendran et al., 1984) e com a motilidade progressiva, como descrito por (Kumi-Diaka e Badtram, 1994; Rodriguez-Gil et al., 1994). Sabe-se que lesões na membrana plasmática podem não refletir diretamente em algumas características seminais, porém, inviabilizam a célula por alterar a funcionalidade da membrana interferindo no processo de fertilização (Kumi-Diaka e Badtram,1994).

\section{Conclusões}

Conclui-se que os dois sistemas de resfriamento conservam de forma semelhante as características do sêmen por 24 horas, sendo o meio LGM superior ao MMC na manutenção da maioria dos parâmetros seminais.

DOUGLAS-HAMILTON, D. H., OSOL, R., OSOL, G. et al. A field study of the fertility of transported equine semen. Theriogenology, v. 22, n. 3, p. 291-304, 1984.

ENGLAND, G. C. W., ALLEN, W. E. Factors affecting the viability of canine spermatozoa: II. Effects of seminal plasma and blood. Theriogenology, v. 40, n. 2, p. 373-381, 1992.

ENGLAND, G. C. W., PONZIO, P. Comparison of the quality of frozenthawed and cooled-rewarmed dog semen. Theriogenology, v. 46, n. 1, p. 165-171, 1996.

FARSTAD, W. Semen cryopreservation in dogs and foxes. Anim. Reprod. Sci. v. 42, p. 251-260, 1996.

FELDMAN, E.C., NELSON, R.W. Canine and Feline Endocrinology and Reproduction. Philadelphia: W.B. Saunders, 1996. 785 p.

FOOTE, R. H., LEONARD, E. P. The influence of $\mathrm{pH}$, osmotic pressure, glycine, and glycerol on the survival of dog sperm in buffered-yolk extenders. Cornell Vet., v. 54, n.1, p. 78-89, 1964.

HUECK, C. Untersuchugen zur flussigkon - servierung von Pferdesperma unter Verwendung Verchiedener Kuhl und transportsysteme - Labor - studie. Hannover, 1990, 89 p. (Tese de Doutorado) - Hannover: Tierarztlichen Hochschule. 1990.

JEYENDRAN, R. S., VAN DER VEM, H. H., PEREZ-PELAEZ, M. et al. Development of an assay to asses the functional integrity of the human sperm membrane and its relationship to the other semen characteristics. J. Reprod. Fertil. v. 70, p. 219-228, 1984.

KENNEY, R. M., BERGMAN, R. V., COOPER, W. L. et al. Minimal contamination techniques for breeding mares: technique and preliminary findings. Proc. Am. Assoc. Equine Pract. v. 21, p. 327-336, 1975.

KUMI-DIAKA, J. Subjecting canine semen to the hypo-osmotic test. Theriogenology. v. 39, n. 6, p. 1279-1289, 1993. 
KUMI-DIAKA, J., BADTRAM, G. Effect storage on sperm membrane integrity and other functional characteristics of canine spermatozoa: in vitro bioassay for canine semen. Theriogenology, v. 41, n. 7, p.13551366, 1994.

LAGARES, M. A., MEIRELLIS, L. S., WALD, V. B. et al. Efeito de diferentes diluidores sobre a membrana plasmática do espermatozóide eqüino e fertilidade do sêmen resfriado. R. Bras. Ci. Vet. v. 7, n. 3, p.153-156, 2000.

LINDE-FORSBERG, C. Artificial insemination with fresh, chilled extended and frozen-thawed semen in the dog. Vet. Med. Surg. Small Anim. v. 10, n. 1, p. 48-58. 1995.

LOOMIS, P.R. Factors affecting the success of artificial insemination with cooled, transported semen. Proc. Am. Assoc. Equine Pract. v. 38, p. 629-647. 1992.

MAGNAGO, L. G. P. Avaliação física e morfológica do sêmen de cães da raça pastor alemão resfriado a $5^{\circ} \mathrm{C}$. Belo Horizonte, 2000, $79 \mathrm{p}$. Dissertação (Mestrado em Medicina Veterinária) - Escola de Veterinária, Universidade Federal de Minas Gerais, Belo Horizonte, 2000.

MILWARD, C. O. Efeito do resfriamento a $4^{\circ} \mathrm{C}$, sobre as características físicas e morfológicas do sêmen de cães, diluído em meio tamponado de leite desnatado e glicose durante 48 horas e mantido em container para transporte. Niterói, 2002, 46 p. Dissertação (Mestrado em Medicina Veterinária) - Faculdade de Veterinária, Universidade Federal Fluminense, Niterói, 2002.

NISHIKAWA, Y., WIDE, Y. Studies on artificial insemination in the horse.III. A new method of preserving horse spermatozoa, the so-called Baken method. Anim. Breed. Abstr., v. 19, n. 1, Abstr. 53, 1951.
PALMER, E. Factors affecting stallion semen survival and fertility. In: International Congress on Animal Reproduction and Artificial Insemination. Proceedings. Urbana, v. 3, p. 377, 1974.

PICKETT, B. W. Seminal extenders and cooled semen. In: MCKINNON, A. O.; VOSS, J.L. Equine Reproduction. Philadelphia: Lea e Febiger, p. 746-754, 1993.

RODRIGUEZ-GIL, J. E., MONTSERRAT, A., RIGAU, T. Effects of hypo osmotic incubation on acrosome and tail structure on canine spermatozoa. Theriogenology, v. 42, n. 5, p. 815-829, 1994.

ROTA, A., STRÖM, B., LINDE-FORSBERG, C. Effects of seminal plasma and three extenders on canine semen stored at $4^{\circ} \mathrm{C}$. Theriogenology. v. 44, n. 6, p. 885-900, 1995.

SANTOS, I. W., LIMA, V .F .M .H., BINSFELD, L. C., et al. Congelação do sêmen canino comparando diferentes concentrações de glicerol e diferentes tempos de equilíbrio. Arch. Veter. Sci. v. 8, n. 2, p. 57-62, 2003.

SILVA, A. R., SATZINGER, S., LEITE, L. M., SILVA, L. D. M. Gestação obtida por inseminação artificial com sêmen canino refrigerado transportado à distância - relato de caso. Clínica Veterinária, n. 5, p. 56-66, maio/jun. 2004.

SILVA FILHO, J. M. Avaliação do manejo reprodutivo e do sêmen na inseminação artificial de eqüinos. 1994, 408 p. Tese (Doutorado em Zootecnia) - Faculdade de Veterinária, Universidade Federal de Viçosa, Viçosa, MG, 1994.

VAN DER HOLST, W. Stallion semen production in A.I programs in the Netherlands. In: The Male in farm animal reproduction. Boston: Courot, M. (Ed.) p. 195-201,1984.

WATSON, P. F. The roles of lipid and protein in the protection of ram spermatozoa at $5^{\circ} \mathrm{C}$ by egg-yolk lipoprotein. J. Repr. Fert., v. 62, p. 483-492, 1981. 